\title{
Identification of core genes and outcome in gastric cancer using bioinformatics analysis
}

\author{
Chenhua Sun ${ }^{1, *}$, Qi Yuan ${ }^{2, *}$, Dongdong Wu ${ }^{1}$, Xiaohu Meng ${ }^{1}$ and Baolin Wang ${ }^{1,3}$ \\ ${ }^{1}$ The Second Clinical Medical College of Nanjing Medical University, Nanjing, Jiangsu, China \\ ${ }^{2}$ Department of Endocrinology, Huai'an First People's Hospital, Nanjing Medical University, Huai'an, Jiangsu, China \\ ${ }^{3}$ Department of General Surgery, The Second Affiliated Hospital of Nanjing Medical University, Nanjing, Jiangsu, China \\ *These authors contributed equally to this work and co-first authors
}

Correspondence to: Baolin Wang, email: wang_blin@163.com

Keywords: gastric cancer, bioinformatics analysis, protein-protein interaction, diagnosis, combination medicine

Received: June 14, $2017 \quad$ Accepted: July 25, $2017 \quad$ Published: August 09, 2017

Copyright: Sun et al. This is an open-access article distributed under the terms of the Creative Commons Attribution License 3.0 (CC BY 3.0), which permits unrestricted use, distribution, and reproduction in any medium, provided the original author and source are credited.

\section{ABSTRACT}

Gastric cancer (GC) is a common malignant neoplasm of gastrointestinal tract. We chose gene expression profile of GSE54129 from GEO database aiming to find key genes during the occurrence and development of GC. 132 samples, including 111 cancer and $\mathbf{2 1}$ normal gastric mucosa epitheliums, were included in this analysis. Differentially expressed genes (DEGs) between GC patients and health people were picked out using GEO2R tool, then we performed gene ontology (GO) analysis and Kyoto Encyclopedia of Gene and Genome (KEGG) pathway analysis using The Database for Annotation, Visualization and Integrated Discovery (DAVID). Moreover, Cytoscape with Search Tool for the Retrieval of Interacting Genes (STRING) and Molecular Complex Detection (MCODE) plug-in was utilized to visualize protein-protein interaction (PPI) of these DEGs. There were 971 DEGs, including 468 up-regulated genes enriched in focal adhesion, ECM-receptor interaction and PI3K-Akt signaling pathway, while 503 down-regulated genes enriched in metabolism of xenbiotics and drug by cytochrome P450, chemical carcinogenesis, retinol metabolism and gastric acid secretion. Three important modules were detected from PPI network using MCODE software. Besides, Fifteen hub genes with high degree of connectivity were selected, including BGN, MMP2, COL1A1, and FN1. Moreover, the Kaplan-Meier analysis for overall survival and correlation analysis were applied among those genes. In conclusion, this bioinformatics analysis demonstrated that DEGs and hub genes, such as BGN, might promote the development of gastric cancer, especially in tumor metastasis. In addition, it could be used as a new biomarker for diagnosis and to guide the combination medicine of gastric cancer.

\section{INTRODUCTION}

Gastric cancer (GC) is one of the most common tumor of gastrointestinal tract. Lately, the therapy of gastric cancer patients depended largely on the pathologic staging, while pathological biopsy still played an important role during the diagnose of GC. However, this method had no effect on healthy people who were potentially becoming GC patients, even caused the patient unnecessary suffering, and most people with specific discomfort symptoms were advanced patients with gastric cancer [1]. So the global five-year survival rate of gastric cancer was only about $10 \%$ [1]. However, in America, this rate was about $30.4 \%$ [2], and 5-year survival was over $65 \%$ in South Korea because of the extensive screening work for gastric cancer [3].

Recently, several biomarkers were used for the screening and diagnosis of gastric cancer. For example, carbohydrate antigen 72-4 (CA72-4) is one of these tumor markers and it can be used as a screening test for 
gastric cancer. CA72-4 was an independent significant prognostic factor for relapse free survival and overall survival of gastric cancer [4]. However, this indicator is overly sensitive and it can be elevated in many types of tumors, such as ovarian carcinoma, lung cancer and pancreatic cancer [5-7]. The sensitivity at the time of primary diagnosis of ovarian carcinoma was up to $47 \%$ for CA72-4 [5]. The area under the curve of CA72-4 was 0.884 for lung cancer [6] and the sensitivity of CA72-4 in pancreatic cancer was $25.5 \%$ [7]. Therefore, it was necessary to investigate the occurrence and development of gastric cancer and detect novel and specific early diagnosis markers.

High throughput sequencing is increasingly being widely used and it has been used as a very significant tool for life sciences, such as cancer early diagnosis, cancer grading and prognosis prediction [8]. In this analysis, we chose GSE54129 from Gene Expression Omnibus (GEO), and used GEO2R online tool to detect the differentially expressed genes (DEGs). Followed by, we established PPI network of the DEGs and picked out hub genes with high degree of connectivity. Moreover, analysis of biological process (BP), molecular function (MF), cellular component (CC) and KEGG pathways of the DEGs and three modules were performed. Besides, overall survival (OS) analysis of these hub genes were made using the Kaplan Meier plotter online database (http://kmplot.com/ analysis/) [9]. Then, the correlation analysis based on TCGA database was performed to visualize the potential relationship between genes, and guide the specific medicine treatment for patients.

\section{RESULTS}

\section{Identification of DEGs and hub genes}

There were 111 gastric cancer samples and 21 normal samples in this study. The GEO2R online analysis tool was applied to detect the DEGs, using adjust $P$ value $<0.05$ and $|\operatorname{logFC}| \geq 2$ as cut-off criteria. A total of 971 differential expressed genes were detected after the analysis of GSE54129; of which, 468 were up-regulated genes and 503 were down-regulated. Besides, 15 hub genes with high degree of connectivity were selected (Table 1).

\section{GO function and KEGG pathway enrichment analysis}

For a more in-depth understanding of the selected DEGs, GO function and KEGG pathway enrichment analysis was applied using DAVID. We imported all DEGs to DAVID software and GO analysis results showed that up-regulated DEGs and down-regulated DEGs were particularly enriched in biological processes (BP), including vasculature development, blood vessel morphogenesis and cardiovascular and circulatory system development for up-regulated DEGs, and for downregulated including epithelial cell differentiation, digestion and epithelium development (Table 2). For molecular function (MF), the upregulated DEGs were enriched in glycosaminoglycan, heparin, sulfur compound, receptor and protein complex binding, and the down-regulated DEGs were enriched in oxidoreductase activity, acting on $\mathrm{CH}-\mathrm{OH}$ group of donors, NAD or NADP as acceptor and retinol dehydrogenase and aldo-keto reductase (NADP) activity (Table 2). In addition, GO cell component (CC) analysis also displayed that the up-regulated DEGs were significantly enriched in the extracellular matrix, proteinaceous extracellular matrix and extracellular region part, and down-regulated DEGs enriched in extracellular region part and apical part of cell (Table 2).

Table 3 showed the most significantly enriched KEGG pathway of the up-regulated and down-regulated DEGs. The up-regulated DEGs were enriched in focal adhesion, ECM-receptor interaction, PI3K-Akt signaling pathway, protein digestion and absorption and vascular smooth muscle contraction, while the down-regulated DEGs were enriched in metabolism of xenbiotics by cytochrome P450, chemical carcinogenesis, retinol metabolism, drug metabolism by cytochrome P450 and gastric acid secretion.

\section{The kaplan meier plotter and expression level of hub genes}

The prognostic information of the 15 hub genes was freely available in http://kmplot.com/analysis/. It was found that expression of BGN (HR 1.9 [1.56-2.32], $P=1.3 \times 10^{-10}$ ) was associated with worse overall survival (OS) for gastric cancer patients, as well as MMP2 (HR 1.78 [1.47-2.16], $P=2.6 \times 10^{-9}$ ), COL1A1 (HR 1.49 [1.22-1.81], $P=8.2 \times 10^{-5}$ ) and FN1 (HR 1.46 1.23-1.74], $P=1.3 \times 10^{-5}$ ) (Figure 3). Then, we used GEPIA to detect the hub genes expression level between cancer and healthy people, and Figure 4A reflected that compared to normal, BGN significantly increased expression levels in cancer patients.

\section{Hub genes and module screening from PPI network}

Based on the information in the STRING protein query from public databases, we made the PPI network of the top 15 hub genes with higher degree of connectivity (Figure 1). We selected ACTA2, BGN, MMP2, THBS1, TGFB1, COL1A1, FN1 and ITCH, which with worse overall survival situation according to Kaplan Meier plotter. Based on the GO function, KEGG pathway analysis and the survival analysis, we found that BGN, MMP2, COL1A1 and FN1 were enriched in extracellular matrix, especially in proteinaceous extracellular matrix. 
Table 1: Top 15 hub genes with higher degree of connectivity

\begin{tabular}{ccc}
\hline Gene & Degree of connectivity & Adjusted $\boldsymbol{P}$ value \\
\hline CTNNB1 & 24 & $3.13 \mathrm{E}-22$ \\
FN1 & 16 & $5.13 \mathrm{E}-21$ \\
FYN & 15 & $1.98 \mathrm{E}-34$ \\
MMP9 & 14 & $2.28 \mathrm{E}-09$ \\
COL1A1 & 11 & $1.47 \mathrm{E}-33$ \\
ITCH & 11 & $1.62 \mathrm{E}-30$ \\
TGFB1 & 10 & $6.43 \mathrm{E}-42$ \\
THBS1 & 9 & $1.03 \mathrm{E}-22$ \\
MMP2 & 9 & $3.07 \mathrm{E}-18$ \\
ACTA2 & 8 & $1.67 \mathrm{E}-08$ \\
ITGA5 & 8 & $5.44 \mathrm{E}-20$ \\
BMP2 & 8 & $5.61 \mathrm{E}-24$ \\
BGN & 7 & $1.50 \mathrm{E}-28$ \\
HSPA4 & 7 & $7.44 \mathrm{E}-32$ \\
PIK3R1 & 7 & $2.29 \mathrm{E}-37$ \\
\hline
\end{tabular}

In order to detect significant modules in this PPI network, we used MCODE plug-in. The top 3 modules were selected (Figure 2). KEGG pathway enrichment analysis showed that these three modules were mainly associated with focal adhesion, PI3K-Akt signaling pathway and ECM-receptor interaction (Figure 2).

\section{DISCUSSION}

Although the decline trends of the GC morbidity have been noted in recent years, it is still the second leading cause of cancer deaths in China [10]. It is mainly because of failure to early screening and diagnosis.

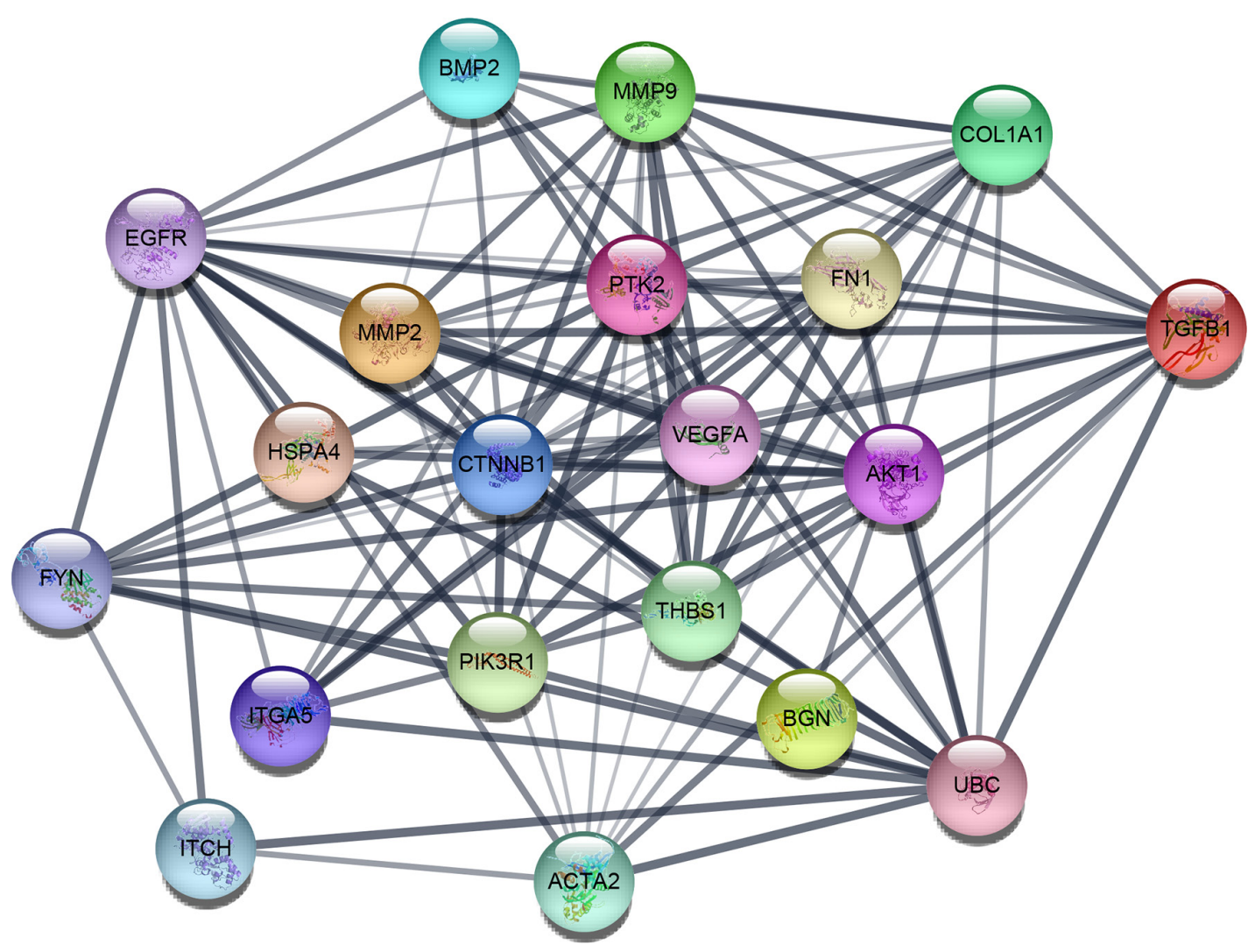

Figure 1: The protein-protein interaction network of top 15 hub genes. 
Table 2: Gene ontology analysis of differentially expressed genes associated with gastric cancer

\begin{tabular}{|c|c|c|c|c|c|c|}
\hline Expression & Category & Term & Count & $\%$ & $P$-Value & FDR \\
\hline \multirow{14}{*}{ Up-regulated } & GOTERM_BP_FAT & GO:0001944 vasculature development & 67 & 19.65 & $7.50 \mathrm{E}-31$ & $1.43 \mathrm{E}-27$ \\
\hline & GOTERM_BP_FAT & GO:0001568 blood vessel development & 62 & 18.18 & $5.72 \mathrm{E}-28$ & $1.09 \mathrm{E}-24$ \\
\hline & GOTERM_BP_FAT & GO:0048514 blood vessel morphogenesis & 56 & 16.42 & $2.00 \mathrm{E}-26$ & $3.82 \mathrm{E}-23$ \\
\hline & GOTERM_BP_FAT & GO:0072358 cardiovascular system development & 75 & 21.99 & $1.28 \mathrm{E}-25$ & $2.43 \mathrm{E}-22$ \\
\hline & GOTERM_BP_FAT & GO:0072359 circulatory system development & 75 & 21.99 & $1.28 \mathrm{E}-25$ & $2.43 \mathrm{E}-22$ \\
\hline & GOTERM_MF_FAT & GO:0005539 glycosaminoglycan binding & 28 & 8.21 & $3.99 \mathrm{E}-15$ & $6.14 \mathrm{E}-12$ \\
\hline & GOTERM_MF_FAT & GO:0008201 heparin binding & 23 & 6.74 & $4.66 \mathrm{E}-13$ & $7.16 \mathrm{E}-10$ \\
\hline & GOTERM_MF_FAT & GO:1901681 sulfur compound binding & 26 & 7.62 & $3.41 \mathrm{E}-12$ & $5.23 \mathrm{E}-09$ \\
\hline & GOTERM_MF_FAT & GO:0005102 receptor binding & 64 & 18.77 & $6.71 \mathrm{E}-10$ & $1.03 \mathrm{E}-06$ \\
\hline & GOTERM_MF_FAT & GO:0032403 protein complex binding & 42 & 12.32 & 2.71E-09 & 4.17E-06 \\
\hline & GOTERM_CC_FAT & GO:0031012 extracellular matrix & 66 & 19.35 & $8.53 \mathrm{E}-32$ & $1.23 \mathrm{E}-28$ \\
\hline & GOTERM_CC_FAT & GO:0005578 proteinaceous extracellular matrix & 52 & 15.25 & 7.99E-28 & $1.16 \mathrm{E}-24$ \\
\hline & GOTERM_CC_FAT & GO:0044421 extracellular region part & 159 & 46.63 & 7.12E-21 & $1.03 \mathrm{E}-17$ \\
\hline & GOTERM_CC_FAT & GO:0005615 extracellular space & 87 & 25.51 & $9.70 \mathrm{E}-20$ & $1.40 \mathrm{E}-16$ \\
\hline \multirow{16}{*}{$\begin{array}{l}\text { Down- } \\
\text { regulated }\end{array}$} & GOTERM_CC_FAT & GO:0005576 extracellular region & 171 & 50.15 & 4.15E-18 & $6.00 \mathrm{E}-15$ \\
\hline & GOTERM_BP_FAT & GO:0030855 epithelial cell differentiation & 42 & 10.85 & $2.99 \mathrm{E}-13$ & $5.61 \mathrm{E}-10$ \\
\hline & GOTERM_BP_FAT & GO:0007586 digestion & 21 & 5.43 & $5.83 \mathrm{E}-11$ & $1.10 \mathrm{E}-07$ \\
\hline & GOTERM_BP_FAT & GO:0060429 epithelium development & 49 & 12.66 & $3.66 \mathrm{E}-08$ & $6.88 \mathrm{E}-05$ \\
\hline & GOTERM_BP_FAT & GO:0006805 xenobiotic metabolic process & 14 & 3.62 & $5.49 \mathrm{E}-08$ & $1.03 \mathrm{E}-04$ \\
\hline & GOTERM_BP_FAT & GO:0006629 lipid metabolic process & 56 & 14.47 & $7.06 \mathrm{E}-08$ & $1.33 \mathrm{E}-04$ \\
\hline & GOTERM_MF_FAT & $\begin{array}{l}\text { GO:0016614 oxidoreductase activity, acting on } \mathrm{CH}-\mathrm{OH} \\
\text { group of donors }\end{array}$ & 22 & 5.68 & $2.20 \mathrm{E}-13$ & $3.43 \mathrm{E}-10$ \\
\hline & GOTERM_MF_FAT & $\begin{array}{l}\text { GO:0016616 oxidoreductase activity, acting on the CH- } \\
\text { OH group of donors, NAD or NADP as acceptor }\end{array}$ & 17 & 4.39 & $9.66 \mathrm{E}-10$ & $1.51 \mathrm{E}-06$ \\
\hline & GOTERM_MF_FAT & GO:0004745 retinol dehydrogenase activity & 8 & 2.07 & 2.60E-08 & 4.05E-05 \\
\hline & GOTERM_MF_FAT & GO:0004033 aldo-keto reductase (NADP) activity & 7 & 1.81 & $1.08 \mathrm{E}-05$ & 0.016818 \\
\hline & GOTERM_MF_FAT & $\begin{array}{l}\text { GO:0016709 oxidoreductase activity, acting on paired } \\
\text { donors, with incorporation or reduction of molecular } \\
\text { oxygen, NAD }(\mathrm{P}) \mathrm{H} \text { as one donor, and incorporation of } \\
\text { one atom of oxygen }\end{array}$ & 7 & 1.81 & $1.71 \mathrm{E}-04$ & 0.266413 \\
\hline & GOTERM_CC_FAT & GO:0044421 extracellular region part & 124 & 32.04 & 5.03E-08 & 7.04E-05 \\
\hline & GOTERM_CC_FAT & GO:0005576 extracellular region & 140 & 36.18 & $1.05 \mathrm{E}-07$ & $1.47 \mathrm{E}-04$ \\
\hline & GOTERM_CC_FAT & GO:0045177 apical part of cell & 26 & 6.72 & $1.38 \mathrm{E}-07$ & $1.93 \mathrm{E}-04$ \\
\hline & GOTERM_CC_FAT & GO:0070062 extracellular exosome & 92 & 23.77 & $3.26 \mathrm{E}-06$ & 0.004567 \\
\hline & GOTERM CC FAT & GO:1903561 extracellular vesicle & 92 & 23.77 & 4.04E-06 & 0.005651 \\
\hline
\end{tabular}

GO: Gene Ontology; FDR: False Discovery Rate.

Therefore, sensitive and specific biomarkers of gastric cancer are urgently needed to be detected.

In this analysis, 111 gastric cancer samples and 21 normal samples were included from the GEO database of GSE54129. A total of 971 DEGs were screened, including 468 up-regulated genes and 503 down-regulated genes. For a more in-depth understanding of these DEGs, we performed GO function and KEGG pathway analysis of these DEGs.

The GO analysis showed that up-regulated DEGs were mainly involved in vessel morphogenesis, glycosaminoglycan binding and extracellular matrix, and down-regulated DEGs were involved in epithelial cell differentiation, oxidoreductase activity, acting on $\mathrm{CH}-\mathrm{OH}$ group of donors, extracellular region part and digestion. Furthermore, the KEGG pathways of up-regulated DEGs included focal adhesion, ECM-receptor interaction, PI3KAkt signaling pathway, protein digestion and absorption and vascular smooth muscle contraction, while the down-regulated DEGs were enriched in metabolism of xenbiotics by cytochrome P450, chemical carcinogenesis, retinol metabolism, drug metabolism by cytochrome P450 and gastric acid secretion. Among these DEGs, 15 hub genes with high degree of connectivity were selected 


\begin{tabular}{|c|c|c|c|c|c|c|}
\hline Category & Term & Count & $\%$ & $P$-Value & Genes & FDR \\
\hline \multirow{5}{*}{$\begin{array}{l}\text { Up- } \\
\text { regulated } \\
\text { DEGs }\end{array}$} & hsa04510:Focal adhesion & 25 & 7.3 & $5.06 \mathrm{E}-11$ & $\begin{array}{l}\text { TLN1, TNC, MYL9, COL6A3, COL6A2, } \\
\text { COL6A1, ZYX, THBS1, COL11A1, } \\
\text { THBS2, PIK3R1, SPP1, THBS4, FN1, } \\
\text { COL4A2, COL4A1, IGF1, FLNA, } \\
\text { VEGFC, FYN, ITGA5, ITGA7, COL1A2, } \\
\text { COL1A1, MYLK }\end{array}$ & $6.32 \mathrm{E}-08$ \\
\hline & $\begin{array}{l}\text { hsa04512:ECM-receptor } \\
\text { interaction }\end{array}$ & 16 & 4.7 & 1.13E-09 & $\begin{array}{l}\text { COL4A2, COL4A1, TNC, ITGA5, } \\
\text { ITGA7, COL6A3, COL6A2, COL1A2, } \\
\text { COL6A1, COL1A1, THBS1, THBS2, } \\
\text { COL11A1, SPP1, THBS4, FN1 }\end{array}$ & $1.42 \mathrm{E}-06$ \\
\hline & $\begin{array}{c}\text { hsa04151:PI3K-Akt signaling } \\
\text { pathway }\end{array}$ & 25 & 7.3 & $1.34 \mathrm{E}-06$ & $\begin{array}{l}\text { MCL1, OSMR, TNC, BCL2L1, IL7R, } \\
\text { COL6A3, COL6A2, COL6A1, IL2RG, } \\
\text { THBS1, COL11A1, THBS2, PIK3R1, } \\
\text { SPP1, THBS4, FN1, COL4A2, COL4A1, } \\
\text { IGF1, YWHAE, VEGFC, ITGA5, } \\
\text { ITGA7, COL1A2, COL1A1 }\end{array}$ & 0.001667 \\
\hline & $\begin{array}{l}\text { hsa04974:Protein digestion and } \\
\text { absorption }\end{array}$ & 12 & 3.5 & $5.64 \mathrm{E}-06$ & $\begin{array}{lcl}\text { COL4A2, } & \text { COL14A1, } & \text { COL4A1, } \\
\text { COL6A3, ELN, COL1A2, } & \text { COL6A2, } \\
\text { COL12A1, COL6A1, } & \text { COL1A1, } \\
\text { COL11A1, COL10A1 } & \end{array}$ & 0.007038 \\
\hline & $\begin{array}{l}\text { hsa04270: Vascular smooth } \\
\text { muscle contraction }\end{array}$ & 12 & 3.5 & 9.93E-05 & $\begin{array}{l}\text { EDNRA, GNA13, ACTG2, ACTA2, } \\
\text { CALD1, PLA2G2A, GUCY1A3, } \\
\text { GUCY1B3, CALCRL, } \\
\text { KCNMB1, MYL9 }\end{array}$ & 0.123856 \\
\hline \multirow{5}{*}{$\begin{array}{l}\text { Down- } \\
\text { regulated } \\
\text { DEGs }\end{array}$} & $\begin{array}{l}\text { hsa00980: Metabolism of } \\
\text { xenobiotics by cytochrome } \\
\text { P450 }\end{array}$ & 12 & 3.1 & $2.08 \mathrm{E}-07$ & $\begin{array}{l}\text { GSTA1, CYP3A4, CYP3A5, CBR1, } \\
\text { CYP2C9, ADH1C, AKR7A3, ADH1A, } \\
\text { ADH7, UGT2B15, AKR1C1, ALDH3A1 }\end{array}$ & $2.56 \mathrm{E}-04$ \\
\hline & $\begin{array}{l}\text { hsa } 05204: \text { Chemical } \\
\text { carcinogenesis }\end{array}$ & 12 & 3.1 & 4.70E-07 & $\begin{array}{l}\text { GSTA1, CYP3A4, CYP3A5, CBR1, } \\
\text { CYP2C19, CYP2C9, CYP2C18, ADH1C, } \\
\text { ADH1A, ADH7, UGT2B15, ALDH3A1 }\end{array}$ & $5.78 \mathrm{E}-04$ \\
\hline & hsa00830:Retinol metabolism & 11 & 2.8 & 5.47E-07 & $\begin{array}{l}\text { CYP3A4, RDH12, CYP3A5, CYP2C9, } \\
\text { CYP2C18, ADH1C, DHRS9, SDR16C5, } \\
\text { ADH1A, ADH7, UGT2B15 }\end{array}$ & $6.72 \mathrm{E}-04$ \\
\hline & $\begin{array}{l}\text { hsa00982:Drug metabolism - } \\
\text { cytochrome P450 }\end{array}$ & 11 & 2.8 & 8.44E-07 & $\begin{array}{l}\text { GSTA1, CYP3A4, FMO5, CYP3A5, } \\
\text { CYP2C19, CYP2C9, ADH1C, ADH1A, } \\
\text { ADH7, UGT2B15, ALDH3A1 }\end{array}$ & 0.001037 \\
\hline & $\begin{array}{l}\text { hsa04971:Gastric acid } \\
\text { secretion }\end{array}$ & 11 & 2.8 & $1.66 \mathrm{E}-06$ & $\begin{array}{l}\text { KCNJ16, KCNJ15, ATP4A, GNAQ, } \\
\text { ATP4B, SLC26A7, KCNE2, GAST, CA2, } \\
\text { SST, KCNK10 }\end{array}$ & 0.002035 \\
\hline
\end{tabular}

KEGG: Kyoto Encyclopedia of Genes and Genomes; FDR: False Discovery Rate.

in the PPI network. Four hub genes with worse overall survival (OS) of gastric cancer patients were detected and the Kaplan Meier-plotter was applied to visualize them, including BGN, MMP2, COL1A1 and FN1. Those four hub genes were enriched in extracellular matrix, especially in proteinaceous extracellular matrix. A recent study reported that extracellular matrix could play a vital role in breast cancer metastasis [11]. We could hypothesize that those four genes might contribute to the metastasis of gastric cancer,

BGN, encoded by this gene was a small cell or cell surrounding proteoglycans, which were structurally closely related to two other small proteoglycans, core proteoglycans and fibrin regulatory proteins. BGN took part in blood vessel remodeling, extracellular matrix organization and carbohydrate metabolic process. It exists 
in the extracellular exosome and this cell component had an inseparable relationship with ACAT1 [12], which inhibited cholesterol esterification in T cells, leading to potentiated effector function and enhanced proliferation of $\mathrm{CD}^{+} \mathrm{T}$ cells [13]. The Figure 4B showed the results of the correlation analysis between BGN and ACAT1. BGN and ACAT1 are obviously positively correlated. A combined therapy of the ACAT inhibitor avasimibe plus an anti-PD-1 antibody showed a delightful effect of suppressing the tumor development and progression [13], which provided a whole new perspective for us to perform medical work, using specific tumor inhibitor of BGN and an anti-PD-1 antibody to fight with carcinoma.

Matrix metallopeptidase 2 (MMP2), a member of MMP family which were zinc-dependent enzymes capable of cleaving components of molecules and the extracellular matrix involved in signal transduction. Unlike most MMP family members, activation of MMP2 could happen on the cell membrane, and it could be activated by proteases, or with no requirement for proteolytical removal of the pro-domain by its S-glutathiolation. MMP2 was involved in many pathways in cancer, and it existed in many proteoglycans in cancer. Moreover, the expression level of MMP2 in sarcoma was much high than that in normal tissue based on TCGA database.
Analysis of the three selected modules from the PPI network showed that gastric cancer was associated with focal adhesion, PI3K-Akt signaling pathway and ECMreceptor interaction. It was reported that high expression of focal adhesion kinase (FAK) activity was associated with elevated level of fibrosis and poor $\mathrm{CD}^{+} \mathrm{T}$ cell infiltration. Focal adhesion kinase inhibition could substantially limit tumor progression and extend the survival time of cancer patients [14]. The top perturbed pathways in gastric cancer included focal adhesion and adherens junction, in which RHOA gene and any other differentially expressed genes we detected participated in these biological processes [15]. Collagen, type I, alpha 1 (COL1A1) participated in the process of focal adhesion, and it could provide a new way for us to perform genome personalized treatment. Several studies indicated that numerous components of the phosphatidylinositol-3-kinase (PI3K)/AKT pathway were targeted by amplification, mutation and translocation more frequently than any other pathway in cancer patients, leading to pathway activation. Fibronectin 1 (FN1), predominantly expressed in various malignancies but not in normal tissues, was mainly involved in this pathway [16] and a specific tumor regimen for the FN1 gene could be implemented. The control of cell growth is increased by a coordinated response to growth factor
A

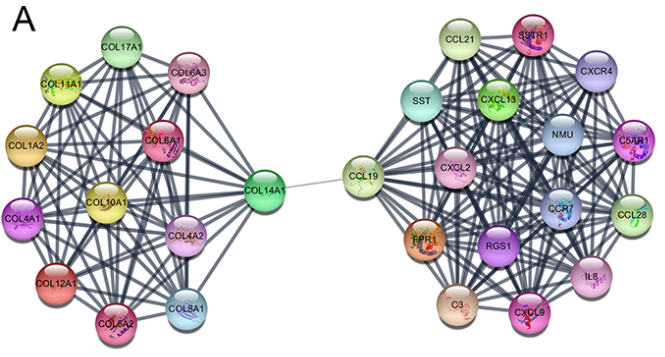

C

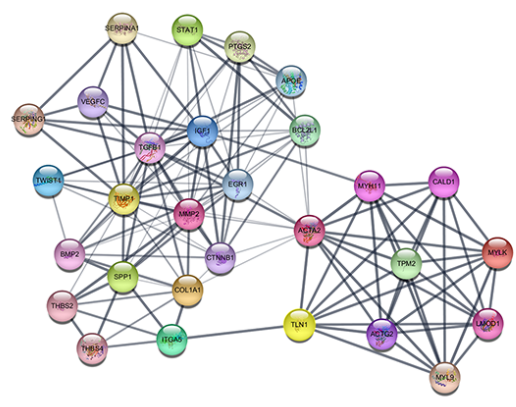

$\mathrm{E}$

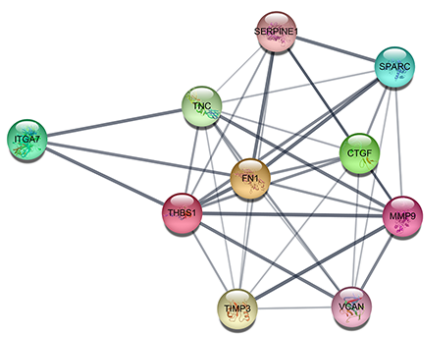

B

\begin{tabular}{|c|c|c|c|}
\hline Term & P-Value & FDR & Genes \\
\hline Protein digestion and absorption & $1.64 \mathrm{E}-14$ & $1.42 \mathrm{E}-11$ & $\begin{array}{l}\text { COL4A2, COL17A1, COL4A1, COL14A1, COL6A3, } \\
1 \text { COL6A2, COL1A2, COL12A1, COL6A1, COL11A1, } \\
\text { COL10A1 }\end{array}$ \\
\hline Chemokine signaling pathway & $3.87 \mathrm{E}-08$ & $3.38 \mathrm{E}-05$ & $\begin{array}{l}\text { CXCR4, CXCL13, CCL21, CXCL2, CXCL9, } \\
9, \text { CCL28 }\end{array}$ \\
\hline ECM-receptor interaction & $1.65 \mathrm{E}-07$ & 1.44E-04 & $\begin{array}{l}\text { COL4A1, COL6A3, COL6A2, COL1A2, } \\
\text { COL11A1 }\end{array}$ \\
\hline Focal achesion & 2.71E-05 & 0.023629 & $\begin{array}{l}9 \text { COL4A2, COL4A1, COL6A3, COL6A2, COL1A2, } \\
\text { COL6A1, COL11A1 }\end{array}$ \\
\hline Cytokine-cytokine receptor interaction & $5.78 \mathrm{E}-05$ & 0.050467 & $\begin{array}{l}7 \text { CCR7, CXCR4, CXCL13, CCL21, CXCL9, CCL19, } \\
\text { CCL28 }\end{array}$ \\
\hline
\end{tabular}

D

\begin{tabular}{lccl}
\hline Term & P-Value & FDR & Genes \\
\hline Focal adhesion & $1.63 E-09$ & $1.81 E-06$ & VEGFC, TLN1, ITGA5, IGF1, COL1A1, THBS2, \\
MYLK, THBS4, CTNNB1, SPP1, MYL9 \\
Pathways in cancer & $6.09 E-05$ & 0.067627 & VEGFC, BMP2, PTGS2, IGF1, BCL2L1, STAT1, \\
& & MMP2, TGFB1, CTNNB1 \\
PI3K-Akt signaling pathway & $1.83 E-04$ & 0.203389 & VEGFC, ITGA5, IGF1, COL1A1, BCL2L1, THBS2, \\
THBS4, SPP1 & \\
$\begin{array}{ll}\text { ECM-receptor interaction } \\
\text { Proteoglycans in cancer }\end{array}$ & $2.98 \mathrm{E}-04$ & 0.330241 & ITGA5, COL1A1, THBS2, THBS4, SPP1 \\
\hline
\end{tabular}

$\mathrm{F}$

\begin{tabular}{lccl}
\hline Term & P-Value & FDR & Genes \\
\hline ECM-receptor interaction & $1.09 \mathrm{E}-04$ & 0.09205 & TNC, ITGA7, THBS1, FN1 \\
MicroRNAs in cancer & $4.38 \mathrm{E}-04$ & 0.369028 & TNC, MMP, THBS1, TIMP3 \\
Proteoglycans in cancer & 0.001179 & 0.990839 & MMP9, THBS1, TIMP3, FN1 \\
Focal adhesion & 0.001402 & 1.177362 & TNC, ITGA7, THBS1, FN1 \\
Pl3K-Akt signaling pathway & 0.005651 & 4.672509 & TNC, ITGA7, THBS1, FN1 \\
\hline
\end{tabular}

Figure 2: Top 3 modules from the protein-protein interaction network. (A) module 1, (B) the enriched pathways of module 1, (C) module 2, (D) the enriched pathways of module 2, (E) module 3, (F) the enriched pathways of module 3. 
and cell-extracellular matrix (ECM) interactions. To be one of the family of ECM receptor, integrin was critical to the coordinated responses [17]. FN1 was also involved in this pathway and the expression level of FN1 was closely associated with tumor growth and metastasis [18].

All in all, our bioinformatics analysis identified DEGs and they might play a central role in the occurrence, development and prognosis of gastric cancer. In this study, a total of 971 DEGs and 15 hub genes were selected, and BGN, MMP2, COL1A1 and FN1 might be the core genes of gastric cancer. In order to get more accurate correlation results, we need to start a series of verification experiments later to confirm the results of this prediction. Anyway, this study could provide some
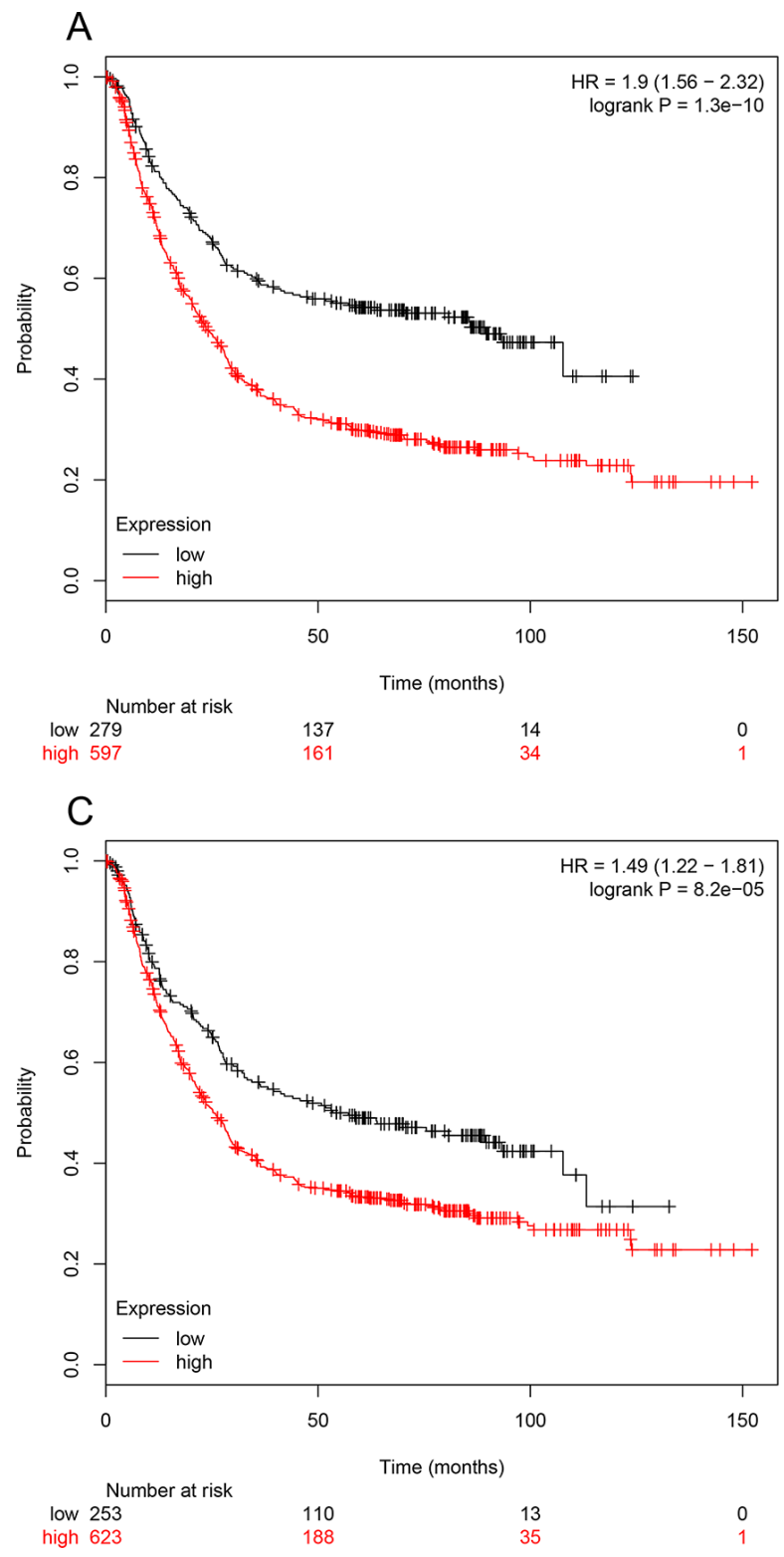

powerful evidence for the future genomic individualized treatment of gastric cancer.

\section{MATERIALS AND METHODS}

\section{Microarray data}

We chose gene expression profile of GSE54129 from GEO database, which was a public and freely available database. GSE54129, which was based on agilent GPL570 platform ([HG-U133_Plus_2] Affymetrix Human Genome U133 Plus 2.0 Array). The GSE54129 dataset included 132 samples, containing 111 gastric cancer samples and 21 normal gastric mucosa epithelium.
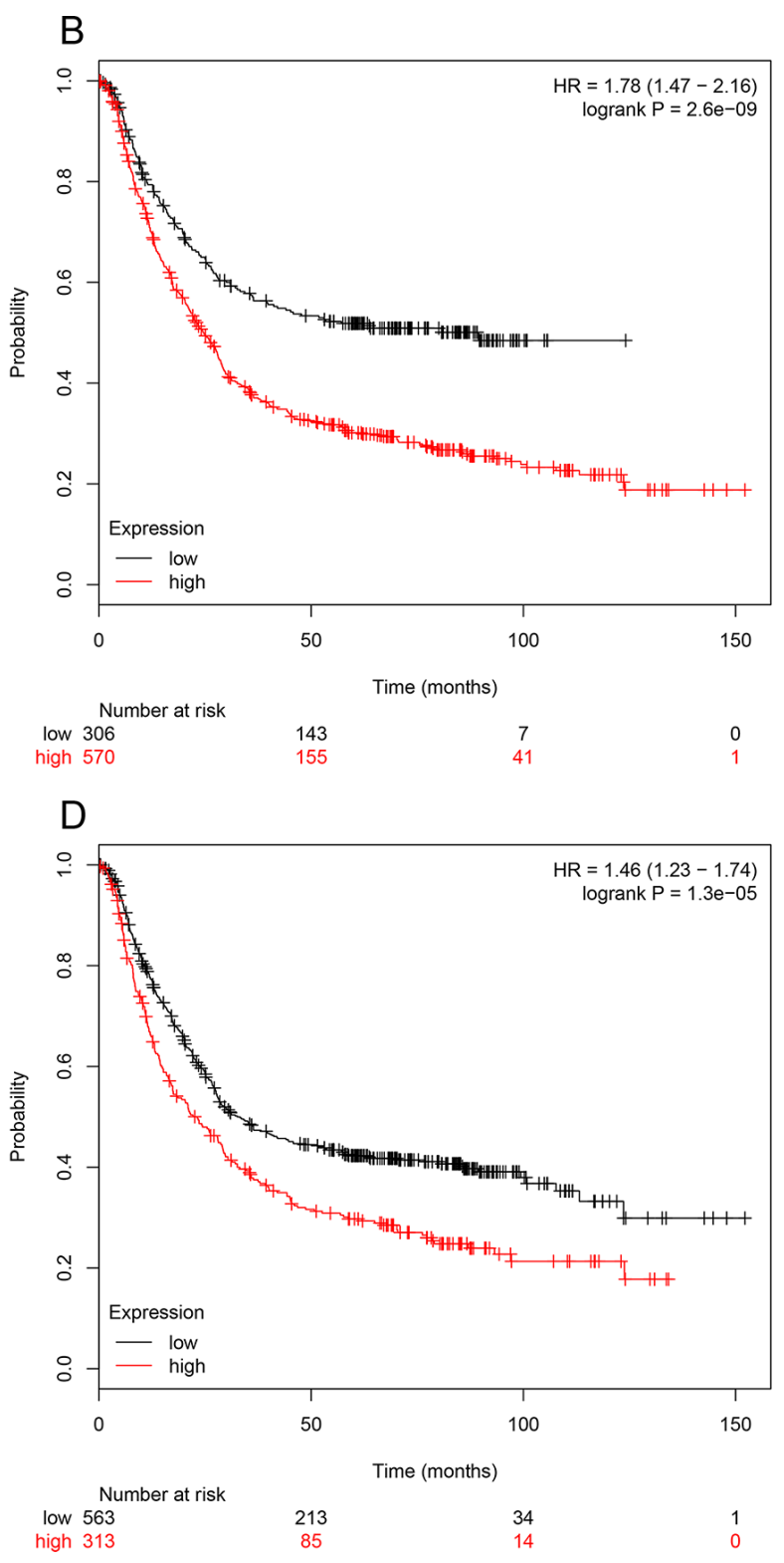

Figure 3: Prognostic value of four genes (BGN (A), MMP2 (B), COL1A1 (C), FN1 (D)) in gastric cancer patients. The desired Affymetrix IDs are valid: 201261_x_at(BGN), 201069_at (MMP2), 202311_s_at (COL1A1), 212464_s_at (FN1). HR: hazard ratio, CI: confidence interval. 
We also downloaded the Series Matrix File of GSE54129 from GEO database.

\section{Data processing of DEGs}

GEO2R (https://www.ncbi.nlm.nih.gov/geo/geo2r/) was applied to detect differentially expressed genes between gastric cancer samples and normal samples [19]. GEO2R is an interactive online tool that allows users to compare two or more groups of samples in a GEO Series and it can analyze most GEO series with gene symbol. The adjust $P$ values were utilized to reduce the false positive rate using Benjamini and Hochberg false discovery rate method by default. The adjust $P$ value $<0.05$ and $|\operatorname{logFC}| \geq 2$ were set as the cut off criterion. Then, 971 DEGs were found, including 468 up-regulated genes and 503 down-regulated genes, and we selected the top 15 genes with high degree of connectivity as hub genes.

\section{Gene ontology and KEGG pathway analysis of DEGs}

Gene ontology analysis (GO) is a common useful method for annotating genes and gene products and for identifying characteristic biological attributes for highthroughput genome or transcriptome data [20]. Kyoto Encyclopedia of Genes and Genomes (KEGG) is a collection of databases dealing with genomes, biological pathways, diseases, drugs, and chemical substances [21]. The Database for Annotation, Visualization and Integrated Discovery (DAVID, https://david.ncifcrf.gov/) is a web- based online bioinformatics resource that aims to provide tools for the functional interpretation of large lists of genes or proteins [22]. $P<0.05$ was set as the cut-off criterion. We could visualize the core biological processes, molecular functions, cellular components and pathways among those DEGs using DAVID.

\section{PPI network and module analysis}

Search Tool for the Retrieval of Interacting Genes (STRING) is online tool designed to evaluate the protein-protein interaction (PPI) information [23]. To detect the potential relationship among those DEGs, we used STRING app in Cytoscape and mapped the DEGs into STRING. And confidence score $\geq 0.4$, maximum number of interactors $=0$ were set as the cut off criterion. Moreover, the Molecular Complex Detection (MCODE) app was utilized to screen modules of PPI network in Cytoscape with degree cutoff $=2$, node score cutoff $=0.2$, $\mathrm{k}$-core $=2$, and $\max$. depth $=100$. The pathway analysis of genes in each module was performed by DAVID. Also, 15 hub genes were also mapped into STRING with confidence score $\geq 0.4$, maximum number of interactors $\leq 5$. GO and KEGG pathway analysis were also made to explore the potential information.

\section{Comparison of the hub genes expression level}

The GEPIA (http://gepia.cancer-pku.cn/index.html) a newly developed interactive web server for analyzing the RNA sequencing expression data of 9,736 tumors
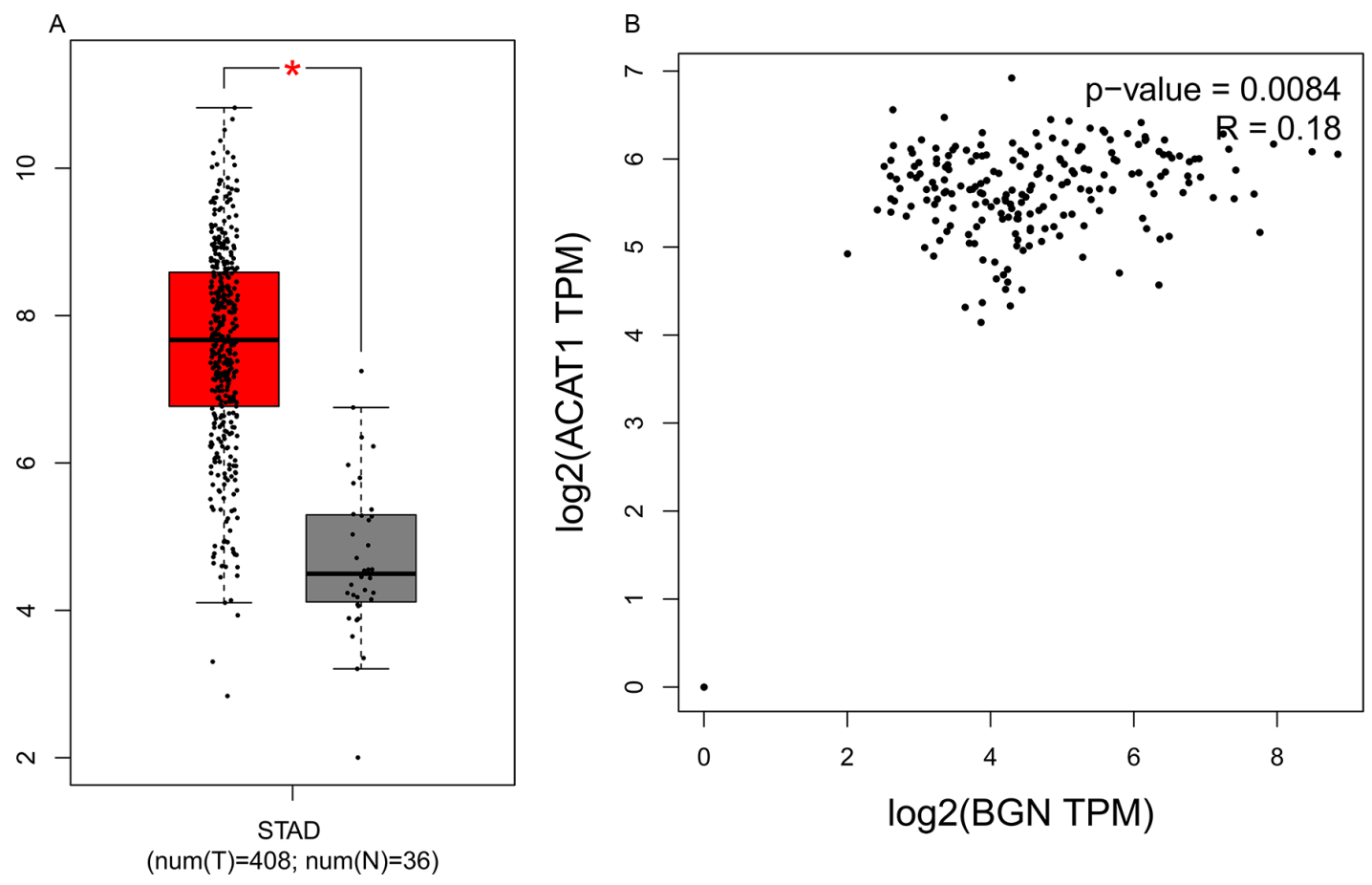

Figure 4: (A) Expression level of BGN in cancer and normal tissues. STAD: Stomach Adenocarcinoma; $* P<0.05$. (B) The correlation analysis between BGN and ACAT1. BGN and ACAT1 are obviously positively correlated. 
and 8,587 normal samples from the TCGA and the GTEx projects, using a standard processing pipeline [24]. It provides customizable functions such as tumor and normal differential expression analysis, and we could demonstrate the expression of hub genes in gastric cancer tissues and normal tissues. Then the boxplot was performed to visualize the relationship.

\section{Survival analysis of hub genes}

Kaplan Meier-plotter (KM plotter, http://kmplot.com/ analysis/) could assess the effect of 54675 genes on survival using 10,461 cancer samples, including 5143 breast, 1816 ovarian, 2437 lung and 1,065 gastric cancer patients with a mean follow-up of 69, 40, 49 and 33 months [9]. The relapse free and overall survival information were based on GEO (Affymetrix microarrays only), EGA and TCGA database. The hazard ratio (HR) with $95 \%$ confidence intervals and $\log$ rank $P$ value were calculated and displayed on the plot.

\section{ACKNOWLEDGMENTS AND FUNDING}

We are grateful to the researchers who provided their data for this analysis, and it is our pleasure to acknowledge their contributions.

\section{CONFLICTS OF INTEREST}

The authors declare that they have no conflicts of interest.

\section{REFERENCES}

1. Orditura M, Galizia G, Sforza V, Gambardella V, Fabozzi A, Laterza MM, Andreozzi F, Ventriglia J, Savastano B, Mabilia A, Lieto E, Ciardiello F, De Vita F. Treatment of gastric cancer. World J Gastroenterol. 2014; 20:1635-49.

2. Howlader N, Noone AM, Krapcho M, Miller D, Bishop K, Altekruse SF, Kosary CL, Yu M, Ruhl J, Tatalovich Z, Mariotto A, Lewis DR, Chen HS, Feuer EJ, Cronin KA. SEER Cancer Statistics Review, 1975-2013, National Cancer Institute. https://seer.cancer.gov/csr/1975_2013/, based on November 2015 SEER data submission, posted to the SEER web site, April 2016.

3. World Cancer Report 2014. World Health Organization. 2014; Chapter 5.4: ISBN 9283204298.

4. Yoon S, Yoo C, Park I, Chang H, Kim T, Lee J, Yook J, Oh S, Kim B, Kang Y. Prognostic significance of preoperative serum tumor markers in the patients with curatively resected advanced gastric cancers. Eur J Cancer, Suppl. 2009; 7:369.

5. Hasholzner U, Baumgartner L, Stieber P, Meier W, Reiter W, Pahl H, Fateh-Moghadam A. Clinical significance of the tumour markers CA 125 II and CA 72-4 in ovarian carcinoma. Int J Cancer. 1996; 69:329-34.
6. Miédougé M, Rouzaud P, Salama G, Pujazon MC, Vincent C, Mauduyt MA, Reyre J, Carles P, Serre G. Evaluation of seven tumour markers in pleural fluid for the diagnosis of malignant effusions. Br J Cancer. 1999; 81:1059-65.

7. Luo G, Liu C, Guo M, Cheng H, Lu Y, Jin K, Liu L, Long J, Xu J, Lu R, Ni Q, Yu X. Potential Biomarkers in Lewis Negative Patients With Pancreatic Cancer. Ann Surg. 2017; 265:800-05.

8. Kulasingam V, Diamandis EP. Strategies for discovering novel cancer biomarkers through utilization of emerging technologies. Nat Clin Pract Oncol. 2008; 5:588-99.

9. Szász AM, Lánczky A, Nagy Á, Förster S, Hark K, Green JE, Boussioutas A, Busuttil R, Szabó A, Győrffy B. Cross-validation of survival associated biomarkers in gastric cancer using transcriptomic data of 1,065 patients. Oncotarget. 2016; 7:49322-33. https://doi.org/10.18632/ oncotarget.10337.

10. Chen W, Zheng R, Baade PD, Zhang S, Zeng H, Bray F, Jemal A, Yu XQ, He J. Cancer statistics in China, 2015. CA Cancer J Clin. 2016; 66:115-32.

11. Chowdhury N, Sapru S. Association of Protein Translation and Extracellular Matrix Gene Sets with Breast Cancer Metastasis: Findings Uncovered on Analysis of Multiple Publicly Available Datasets Using Individual Patient Data Approach. PLoS One. 2015; 10:e0129610.

12. Gonzales PA, Pisitkun T, Hoffert JD, Tchapyjnikov D, Star RA, Kleta R, Wang NS, Knepper MA. Large-scale proteomics and phosphoproteomics of urinary exosomes. $\mathrm{J}$ Am Soc Nephrol. 2009; 20:363-79.

13. Yang W, Bai Y, Xiong Y, Zhang J, Chen S, Zheng X, Meng X, Li L, Wang J, Xu C, Yan C, Wang L, Chang CC, et al. Potentiating the antitumour response of $\mathrm{CD} 8(+) \mathrm{T}$ cells by modulating cholesterol metabolism. Nature. 2016; 531:651-55.

14. Jiang $H$, Hegde S. Targeting focal adhesion kinase renders pancreatic cancers responsive to checkpoint immunotherapy. 2016; 22:851-60.

15. Wang K, Yuen ST, Xu J, Lee SP, Yan HH, Shi ST, Siu HC, Deng S, Chu KM, Law S, Chan KH, Chan AS, Tsui WY, et al. Whole-genome sequencing and comprehensive molecular profiling identify new driver mutations in gastric cancer. Nat Genet. 2014; 46:573-82.

16. Xiang L, Xie G, Ou J, Wei X, Pan F, Liang H. The extra domain A of fibronectin increases VEGF-C expression in colorectal carcinoma involving the PI3K/AKT signaling pathway. PLoS One. 2012; 7:e35378.

17. Cambier S, Mu DZ, O’Connell D, Boylen K, Travis W, Liu $\mathrm{WH}$, Broaddus VC, Nishimura SL. A role for the integrin alphavbeta8 in the negative regulation of epithelial cell growth. Cancer Res. 2000; 60:7084-93.

18. Zhang H, Teng X, Liu Z, Zhang L, Liu Z. Gene expression profile analyze the molecular mechanism of $\mathrm{CXCR} 7$ regulating papillary thyroid carcinoma growth and metastasis. J Exp Clin Cancer Res. 2015; 34:16. 
19. Davis S, Meltzer PS. GEOquery: a bridge between the Gene Expression Omnibus (GEO) and BioConductor. Bioinformatics. 2007; 23:1846-47.

20. Ashburner M, Ball CA, Blake JA, Botstein D, Butler H, Cherry JM, Davis AP, Dolinski K, Dwight SS, Eppig JT, Harris MA, Hill DP, Issel-Tarver L, et al, and The Gene Ontology Consortium. Gene ontology: tool for the unification of biology. Nat Genet. 2000; 25:25-29.

21. Kanehisa M, Goto S. KEGG: Kyoto Encyclopedia of Genes and Genomes. Nucleic Acids Research. 2000; 27: 29-34.

22. Huang W, Sherman BT, Lempicki RA. Systematic and integrative analysis of large gene lists using DAVID bioinformatics resources. Nat Protoc. 2009; 4:44-57.
23. Szklarczyk D, Franceschini A, Wyder S, Forslund K, Heller D, Huerta-Cepas J, Simonovic M, Roth A, Santos A, Tsafou KP, Kuhn M, Bork P, Jensen LJ, von Mering C. STRING v10: protein-protein interaction networks, integrated over the tree of life. Nucleic Acids Res. 2015; 43:D447-52.

24. Tang Z, Li C, Kang B, Gao G, Li C, Zhang Z. GEPIA: a web server for cancer and normal gene expression profiling and interactive analyses. Nucleic Acids Res. 2017; 45:W98-102. 